\title{
Natural Interaction for Object Hand-Over
}

\author{
Mamoun Gharbi, Séverin Lemaignan, Jim Mainprice, Rachid Alami \\ CNRS-LAAS, 7 av. du Colonel Roche, F-31077 Toulouse, France \\ Université de Toulouse, UPS, INSA, INP, ISAE, LAAS, F-31077 Toulouse, France \\ Email: surname.name@laas.fr
}

\begin{abstract}
The video presents in a didactic way several abilities and algorithms required to achieve interactive "pick and give" tasks in a human environment. Communication between the human and the robot relies on unconstrained verbal dialogue, the robot uses multi-modal perception to track the human and its environment, and implements real-time 3D motion planning algorithms to achieve collision-free and human-aware interactive manipulation.

We tackle here the issue of natural and legible behaviour for a robot intended to live in domestic environment, amongst human peers. Natural because we aim at unconstrained spoken English mixed with gestures and perspective taking to communicate with the robot; legible because we apply human-aware motion planning algorithms that ensure a smooth human-robot interaction by accounting for implicit social rules which appear in placement or approach strategies.
\end{abstract}

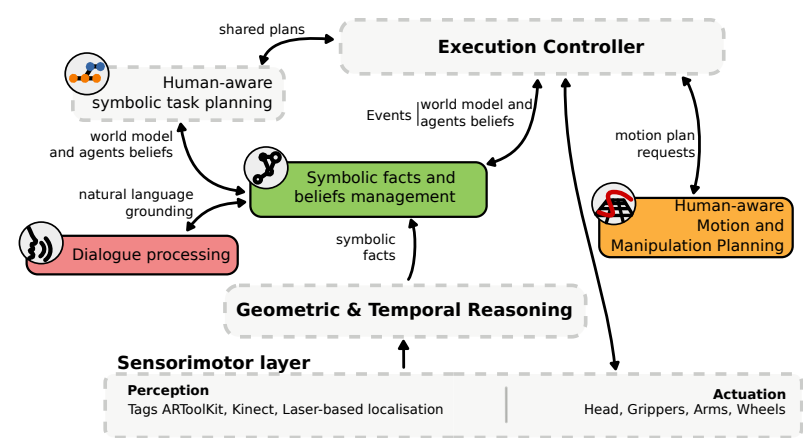

Fig. 1. Overview of the robot deliberative architecture [1]. Coloured modules with plain borders are the specific focus of the video.

\section{A. Modelling of the environment}

The video clip gives a overview of the techniques used online by the robot to build a model of its environment. We rely first on a geometric model, fed by Kinect-like sensors for human tracking and 2D barcodes for object identification and localization.

From this geometric model, we build in real-time a symbolic model, stored as an ontology [2]. This models holds spatial relations between objects and agents, along with a set of affordances (like reachability, visibility, etc.) [3].

\section{B. Natural language processing}

The video also introduces recent work on natural dialogue grounding [3]. The user verbal input is first parsed, then semantically grounded, in tight interaction with the symbolic model. It allows for multi-modal and perspective-aware communication: affordances (like visibility) and human gestures that are recognized (like pointing) and stored in the ontology are used during the grounding process.

\section{Motion planning in human close vicinity}

While planning the robot motions, it is important to account for interaction constraints such as those defined by the proxemics theory. Motion planning techniques such as the ones in [4] enable to account for such constraints using a costbased representation where efficient algorithms incorporating features from stochastic optimization are applied to produce low-cost paths.

D. Sharing effort in handover tasks and proactive robot placement

The planner demonstrated in this video has been introduced in [5], it also accounts for interaction constraints. It consists of sampling handover configurations encoding for the human and robot postures ensuring that they are accessible to them both. The configurations are evaluated regarding an interaction cost and the one that minimizes this cost is returned by the planner. This algorithm enables the robot to proactively decide where to give the object. It can produce plans either for a seated or standing human or through a window as shown in the video. We have introduced a notion of mobility to tune the amount of effort asked to the human.

\section{ACKNOWLEDGMENT}

This work has been supported by EU FP7 "SAPHARI" under grant agreement no. ICT-287513.

\section{REFERENCES}

[1] R. Alami, M. Warnier, J. Guitton, S. Lemaignan, and E. A. Sisbot, "When the robot considers the human..." in Proceedings of the 15th International Symposium on Robotics Research, 2011.

[2] S. Lemaignan, R. Ros, L. Mösenlechner, R. Alami, and M. Beetz, "ORO, a knowledge management platform for cognitive architectures in robotics," in IEEE/RSJ International Conference on Intelligent Robots and Systems, 2010.

[3] S. Lemaignan, R. Ros, E. A. Sisbot, R. Alami, and M. Beetz, "Grounding the interaction: Anchoring situated discourse in everyday human-robot interaction," International Journal of Social Robotics, pp. 1-19, 2011. [Online]. Available: http://dx.doi.org/10.1007/s12369-011-0123-x

[4] J. Mainprice, E. Sisbot, L. Jaillet, J. Cortés, T. Siméon, and R. Alami, "Planning Human-aware motions using a sampling-based costmap planner," in IEEE Int. Conf. Robot. And Autom., 2011.

[5] J. Mainprice, M. Gharbi, T. Siméon, and R. Alami, "Sharing effort in planning human-robot handover tasks," in ROMAN, 2012. 